\title{
La popularité du film nord-américain représente le chinois
}

\author{
Adib Rifqi Setiawan \\ Alobatnis Research Society $(\Lambda \mathrm{RS})$ \\ Pandak, Colo, Kec. Dawe, Kabupaten Kudus, Jawa Tengah, 59353, Indonesia \\ farihaazalea@gmail.com
}

\begin{abstract}
Abstrait
Cet article traite des tropes communs et des représentations du peuple chinois et de la culture chinoise dans un film nord-américain populaire. S'appuyant sur des exemples d'homogénéisation des identités asiatiques (de l'Est, du Sud-Est), de l'appropriation des symboles ou des pratiques culturelles chinoises, du visage jaune comme pratique courante pour jouer des personnages asiatiques, et à la fois de l'hyper-masculinisation et de l'émasculation des hommes chinois, cet article soutient que bien qu'il y ait un lent changement à Hollywood pour s'attaquer à ces problèmes, le contexte du racisme anti-chinois est répandu dans l'industrie cinématographique grand public.
\end{abstract}

Mots - clés : visage jaune, représentation chinoise dans un film, typage, mauvaise interprétation, appropriation culturelle

Cet article narratif discutera de la fausse représentation du peuple chinois dans la culture populaire nord-américaine et analysera des exemples de films pour examiner la manière dont ces fausses déclarations peuvent avoir un impact significatif sur les schémas existants. À l'heure actuelle, le terme «asiatique» est souvent utilisé comme un terme général englobant de nombreux sous-groupes en Asie. Cela pourrait inclure des personnes d'Indonésie, de Corée, d'Iran, d'Inde et de nombreux autres pays, mais les gens en Amérique du Nord supposent généralement que «asiatique» fait référence aux Chinois. En supposant que l'utilisation du terme «asiatique» dans la culture populaire nord-américaine se réfère aux Chinois, d'autres groupes en Asie sont négligés et mal représentés. La représentation du peuple chinois dans la culture populaire peut mettre en évidence les barrières et les luttes auxquelles ils sont confrontés dans les pays occidentaux. Par exemple, On a vu que les caractéristiques de la féminité déformaient le concept d'être une «femme» car elles limitaient leurs options d'expression. En restreignant ses options, le développement d'un individu est limité, et donc opprimé. Le même concept peut être appliqué à ce que la société nord-américaine considère comme «asiatique», puisque les possibilités d'expression d'un individu sont limitées par les fausses déclarations dans la culture populaire, les autres expressions de «l'asiatique» sont effacées.

La représentation chinoise dans la culture populaire nord-américaine a attiré une attention accrue à travers des films comme Crazy Rich Asians (qui se concentrent sur les cultures singapourienne et chinoise), où les peuples d'Asie de l'Est ont vu une augmentation de représentations plus précises dans les films populaires. Cependant, cet article vise à explorer les représentations du peuple chinois qui ont été normalisées dans la culture populaire occidentale: (1) le brouillage d'identités asiatiques distinctes en un Autre homogène et facilement remplaçable; (2) l'appropriation des histoires chinoises racontées à travers le prisme narratif des médias occidentaux; (3) la représentation des hommes chinois comme hyper-masculins ou à travers une lentille émasculée pour correspondre à la perception d'un Autre indésirable; (4) casting d'acteurs blancs pour jouer des personnages asiatiques. 
L'opinion populaire est fortement influencée par les représentations culturelles dans les médias qui dépeignent de manière inexacte la race ou l'ethnicité des gens (Amin-Khan, 2012). Nous pouvons comprendre ces représentations comme la construction de ce que les psychologues appellent des «schémas». Les schémas sont nos représentations mentales qui nous donnent des cadres qui définissent nos idées préconçues pour comprendre les expériences futures. Ces schémas peuvent être modifiés par des expériences, des croyances et des connaissances passées. Les schémas peuvent biaiser nos perceptions de la réalité et des expériences futures parce que nous essayons de les rendre cohérents avec ce que nous savons déjà(Shiraev et Levy, 2016). Pour créer nos schémas, les différences dans d'autres théories sont mises en évidence et nos représentations mentales acceptent des idées qui s'alignent sur les nôtres pour faire avancer notre concept interne(Zhu, 2013).

Comment les fausses déclarations affectent-elles les Chinois d'Amérique du Nord et leur propre perception de leur culture et de leur identité? Alors que la culture chinoise est répandue dans de nombreux contextes médiatiques, il semble y avoir une confusion lorsqu'il s'agit de différencier les différentes cultures «asiatiques» dans les médias. Une erreur courante est de confondre la culture chinoise avec la culture japonaise dans des pièces écrites par des auteurs américains. Les cultures est-asiatiques semblent se mélanger et cela montre un grand malentendu qui se construit avec ces représentations inexactes. De telles fausses déclarations favorisent les malentendus parmi le grand public lorsque le public est exposé à des informations inexactes qui pourraient contribuer à leur schéma existant de ce qu'ils pensent être vrai de la culture chinoise ou de toute culture asiatique.(Chen, 2009).

Dans Crazy Rich Asians, l'actrice Sonoya Mizuno joue un personnage chinois, Araminta Lee. Sonoya est d'origine mi-japonaise et mi-britannique, ce qui crée une représentation inexacte du personnage du film qui induit le public en erreur. La culture normalisée selon laquelle une personne japonaise peut agir comme une personne chinoise, vice versa, ou choisir une personne d'une autre culture asiatique brouille encore davantage les frontières entre les identités asiatiques distinctes et les rend facilement remplaçables par d'autres.

Le film d'animation Disney, Mulan, est un excellent exemple populaire d'appropriation culturelle qui a établi un précédent de la façon dont les valeurs chinoises ont été montrées depuis sa sortie en 1998. Le film dépeint de manière inappropriée des symboles chinois traditionnels pour un effet comique. Par exemple, l'un des personnages est un dragon de la taille d'un lézard nommé Mushu, dont le nom est le même qu'un plat ethniquement chinois. Il serait tout aussi illogique d'avoir un cow-boy nommé «Cornbread» dans un film occidental. Les aspects ethniques du film ont été modifiés pour dépeindre une culture occidentale «dominante» avec des valeurs féministes. De nombreux personnages masculins du film ont été montrés comme cruels et suggéraient une société fortement patriarcale. Pour plaire à un public occidental, des aspects de «l'Autre» ont été mis en évidence pour rendre la culture chinoise plus exotique et différente de la culture occidentale. Le film, qui a été adapté du poème original Ballad of Mulan qui se concentrait sur la loyauté, le féminisme et la piété filiale - un concept dans lequel les aînés sont respectés et soutenus par leurs enfants ou les jeunes générations, est déformé et déformé dans l'adaptation de Disney. Mulan s'est concentré sur l'individualité qui est un concept largement occidental, il souligne que les personnages féminins sont exotiques plutôt que de reconnaître qu'ils étaient des femmes fortes, et décrit la société chinoise comme à l'envers en mettant l'accent sur le patriarcat dans les rôles masculins modifiés.(Yin, 2014). Étant donné que ce film a été commercialisé comme un film pour enfants, il aurait pu avoir un impact important en contribuant à de nombreux schémas de chinois. En modifiant l'accent de l'histoire sur l'individualité de Mulan et sa volonté de se 
libérer de sa famille, cela renforce le fait que les familles chinoises imposent des contraintes à leurs enfants et sont une culture restrictive. Ces changements déforment les histoires chinoises et sont adaptés culturellement pour répondre aux besoins marketing des médias occidentaux en mettant en avant les valeurs occidentales.

Les hommes chinois dans les films hollywoodiens sont souvent décrits comme hypermasculins ou à travers une lentille émasculée pour faire avancer le concept d'altérité. Bruce Lee est un acteur bien connu qui est devenu l'icône de la masculinité chinoise qui serait attrayante pour les protagonistes féminines blanches. Dans d'autres films populaires tels que Sixteen Candles avec le portrait de Long Duk Dong d'un étudiant «asiatique», il est parfois dépeint d'une manière émasculée ou aussi agressif pour correspondre à la perception d'un «Autre» indésirable. La représentation du peuple chinois comme quelqu'un à ridiculiser ou comme dangereux renforce encore l'image des hommes blancs supérieurs à bien des égards. Parmi les autres représentations chinoises de maîtres de kung-fu " cool ", citons Jackie Chan dans l' heure de pointesérie et Jet Li dans de nombreux autres films d'action de la fin des années 1990 au début des années 2000. Bien que ces acteurs masculins aient été qualifiés de héros, il existe encore de nombreux stéréotypes et «blagues» racistes dans les films. Aux heures de pointe, Jackie Chan joue un policier considéré comme un protagoniste, mais il est appelé le «flic Chung King» parce qu'il est chinois. Cela mine la légitimité de son personnage en tant qu'officier de police et affaiblit ses capacités par rapport aux autres officiers non chinois du film. Les «blagues» subtiles qui renforcent les stéréotypes chinois tout au long du film renforcent la suprématie blanche. D'une certaine manière, le portrait de ces hommes chinois et leur masculinité se contredisent puisqu'ils sont d'un côté ce héros cool et combattant mais d'un autre côté, ils sont encore réduits à des " blagues " avec des stéréotypes.(Zhu, 2013).

Les films hollywoodiens ont une histoire de casting d'acteurs qui ne sont pas asiatiques pour jouer des rôles «asiatiques», décrits comme Yellowface, par lesquels des acteurs blancs se maquillent pour imiter des traits «asiatiques» tels que la peau jaune et les yeux plus petits. Un exemple historique bien connu dans le film est la série de films $D r . F u$ Manchu qui a commencé en 1923 et s'est terminée en 1969 avec des acteurs masculins blancs dans Yellowface pour jouer Fu Manchu. Bien que les exemples de Yellowface acceptables dans les médias s'estompent, il a fallu encore près d'un siècle pour que ce changement mineur se produise(Masuchika, 2013). Fantôme dans la coquilleest sorti en 2017 avec Scarlett Johansson comme personnage principal dans la recréation d'un anime japonais du film. Les principaux acteurs de l'anime sont japonais, mais les producteurs ont choisi de jouer Scarlett Johansson en raison de sa popularité en Amérique du Nord et du fait qu'il se serait probablement bien commercialisé. Il y a eu une controverse lors de la planification du film où les gens se sont demandé pourquoi une actrice blanche avait été choisie pour un rôle japonais. Pour tenter de résoudre le problème, la production de Paramount et Dreamworks avait suggéré qu'ils pourraient modifier l'apparence de Scarlett en utilisant des effets numériques pour paraître plus asiatique. Cette «solution» semble être encore plus un pas en arrière et l'idée a finalement été abandonnée. Le film est finalement sorti et a contribué à la représentation raciste de personnages asiatiques dans les médias.

En 2016, Matt Damon était le rôle principal masculin dans La Grande Muraille où il a joué un personnage européen au lieu du film ayant un acteur et un personnage chinois. D'autres rôles masculins de soutien ont été joués par des acteurs chinois bien connus qui auraient pu jouer le rôle principal masculin. Mais encore une fois, pour attirer le marché occidental, il semble qu'un protagoniste masculin blanc était encore nécessaire. Beaucoup ont estimé que 
cette décision de casting avait enlevé de nombreuses opportunités aux acteurs chinois de l'industrie cinématographique et ne permettait pas une représentation précise. La décision de Disney d'avoir Liu Yifei, une actrice sino-américaine, joue Mulan dans leur remake en direct de Mulanpeut être interprété comme une tentative de corriger le blanchiment antérieur de personnages de la culture populaire qui est devenu extrêmement controversé. La nouvelle qu'une actrice chinoise a été choisie pour jouer un rôle chinois a suscité de nombreux retours positifs de la part des téléspectateurs en 2017. Cependant, cette petite victoire soulève la question de savoir pourquoi les gens devraient être soulagés en premier lieu de voir quelqu'un de la même race jouer un personnage conforme à la race de l'acteur. Nous ne devrions pas avoir besoin de célébrer des représentations exactes de la race aujourd'hui.

Parallèlement au changement de représentation fidèle de la race, Hollywood est frappé par le film Crazy Rich Asiansen 2018, cela a changé la vision des personnes «asiatiques» dans la culture populaire. Ce film a une distribution majoritairement chinoise et sud-asiatique et est célébré par beaucoup comme un grand pas en avant dans la rupture des stéréotypes sur les Chinois en ayant des représentations précises des personnes «asiatiques» car la plupart des personnages de l'histoire sont chinois. Bien que les idéaux enracinés des hommes chinois émasculés ou hyper-masculins existent toujours, les femmes trouvent que les hommes «asiatiques» sont moins attirants que l'homme moyen. Cependant, en présentant un Malaisien-Britannique comme le mâle principal dans un rôle romantique, les stéréotypes selon lesquels les hommes «asiatiques» sont indésirables et inintéressants pourraient éventuellement être niés et considérés comme tout aussi attrayants que les hommes blancs. Les représentations de personnages dans un film ne sont qu'une partie de la réalité, si elles contiennent une quelconque vérité, mais est souvent cru par ceux qui ne font pas partie du groupe culturel représenté, surtout s'ils n'ont pas d'exemples réels pour contrer ces représentations. Avec des représentations de plus en plus précises des cultures dans la culture populaire dominante, les individus de la race représentée pourraient se sentir moins pressés et stéréotypés par les représentations inexactes.

À la lumière du casting d'acteurs de la bonne race pour jouer les rôles principaux, Simu Liu a reçu beaucoup de haine lorsqu'il a été choisi pour jouer Shang-Chi dans le film de Marvel Shang-Chi et la légende des dix anneaux. Encore une fois, les effets d'une histoire d'hommes asiatiques émasculants et hyper-masculins sont perçus comme de nombreuses personnes le jugeant «trop laid» pour jouer le rôle du premier super-héros «asiatique» de Marvel. Ces critiques font ressortir les connotations d'attractivité que notre société a construites. Les mesures de l'attractivité suggérées par de nombreuses critiques sont influencées par les normes de beauté eurocentriques et occidentales. Simu Liu s'est publiquement prononcé contre ces critiques et a déclaré qu'il ne permettra pas aux opinions des autres de définir qui il est. Ce dynamisme dont il a fait preuve peut aider les Chinois d'Amérique du Nord à ne pas tenir compte des fausses déclarations dans la culture populaire et à créer leur propre récit précis.

Notre société est fortement influencée par nos histoires qui portent souvent des concepts avec lesquels les gens ne sont pas nécessairement d'accord, mais ils hésitent à changer leurs schémas existants. Avec la lenteur des changements observés au fil des ans, un activisme supplémentaire pour attirer l'attention sur l'homogénéisation des identités asiatiques est nécessaire pour que différents groupes soient reconnus. Il reste encore du travail à faire pour mettre un terme aux stéréotypes culturels, à l'appropriation et au blanchiment dans la culture populaire occidentale par une éducation plus poussée afin de corriger les schémas existants et futurs. 


\section{Reconnaissance}

Cet article est dédié à mon modèle de rôle Laila Fariha Zein, de son incroyable inspiration et de sa motivation, pas pour son infinie façonner mon état d'esprit. Tellement incroyable, fière et chanceuse d'être son mentoré.

\section{Références}

Amin-Khan, T. (2012, October 12). New Orientalism, Securitisation and the Western Media's Incendiary Racism. Third World Quarterly, 33(9), 1595-1610.

Chen, M. (2009, Spring). Seeking Accurate Cultural Representation: Mahjong, World War II, and Ethnic Chinese in Multicultural Youth Literature. Multicultural Education, 16(3), 2-10.

Masuchika, G. N. (2013, January 18). "Yellowface" in movies: a survey of American academic collections. Collection Building, 32(1), 31-36.

Shiraev, E., \& Levy, D. (2016). Cross-Cultural Psychology: Critical Thinking and Contemporary Applications, Sixth Edition (6th ed.). Abingdon: Routledge.

Yin, J. (2014, November 10). Popular Culture and Public Imaginary: Disney Vs. Chinese Stories of Mulan. Javnost - The Public, 18(1), 53-74.

Zhu, Z. (2013, January 30). Romancing 'kung fu master'-from 'yellow peril'to 'yellow prowess'. Asian Journal of Communication, 23(4), 403-419. 